\title{
MHD NUMERICAL SIMULATIONS OF MAGNETIC RECONNEC- TION ASSOCIATED WITH EMERGING FLUX
}

\author{
K. SHIBATA ${ }^{1}$, S. NOZAWA ${ }^{2}$, and R. MATSUMOTO ${ }^{3}$ \\ ${ }^{1}$ National Astronomical Observatory, Mitaka, Tokyo 181, Japan \\ 2 STE Laboratory, Nagoya University, Toyokawa 442 \\ ${ }^{3}$ College of Arts and Sciences, Chiba University, Chiba 260
}

\begin{abstract}
Two-dimensional (2D) magnetohydrodynamic (MHD) numerical simulations have been performed to study magnetic reconnection between emerging flux and the overlying coronal magnetic field, taking into account of gravity. It is found that (1) reconnection starts when most of chromospheric mass in the current sheet between the emerging flux and the coronal field has drained down along the loop because of gravity, (2) multiple magnetic islands, which confine cool, dense chromospheric plasma, are created in the sheet; the islands coalesce dynamically and are ejected along the sheet, together with the ambient hot plasma, at Alfven speed. The coexistence of hot and cool plasmas in the mass ejection (jet) associated with the reconnection seems to explain those $\mathrm{X}$-ray jets observed by Yohkoh, which are identified with $\mathrm{H} \alpha$ surges.
\end{abstract}

\section{INTRODUCTION}

Compact flares and X-ray bright points are often associated with the emergence of new magnetic flux from below the photosphere (e.g., Kundu and Woodgate 1986). To explain these phenomena associated with emerging flux, models including a current sheet between the emerging flux and the overlying coronal and/or chromospheric magnetic field have been proposed (e.g., Forbes and Priest 1984). None of the previous emerging flux models, however, took into account the effect of gravity which is essential to model the emerging flux because the force carrying the magnetic flux into the atmosphere is the magnetic buoyancy.

Here we give a brief report of the results of two-dimensional (2D) magnetohydrodynamic (MHD) numerical simulations of the magnetic reconnection between emerging flux and the coronal field, taking into account the effect of gravity. (See Shibata et al. 1992a for a more detailed discussion.) This is a direct extention of our previous numerical simulations of emerging flux (Shibata et al. 1989, 1990; Nozawa et al. 1992).

\section{NUMERICAL SIMULATIONS}

We simulate emerging flux using numerical solutions of the nonlinear, time dependent, resistive MHD equations in a 2D Cartesian geometry, taking into account the gravitational acceleration, $g$, in the negative $z$-direction (Shibata et al. 1989, 1990). The 
initial model consists of a magnetostatic layer composed of a convectively stable twotemperature gas layer in the upper region (as a simplified model of the solar corona and chromosphere/photosphere) with a convectively unstable layer in the lower region (as a model convection zone). The units of length, velocity, and time in the simulation are $H, C_{s}$ and $H / C_{s}$, respectively, where $C_{s}$ and $H$ are the sound speed and the pressure scale height in the photosphere/chromosphere, respectively. When we compare numerical results with observations, we will use $H=200 \mathrm{~km}, C_{s}=10 \mathrm{~km} \mathrm{~s}^{-1}, \tau=H / C_{s}=20$ s. The initial magnetic field is horizontal (in the $x$-direction), and is localized in the convection zone, while in the corona it is nearly uniform and antiparallel to the convection zone magnetic flux. There is initially no magnetic field between the convection zone and the corona (see $t=0$ of Fig. 1). The localized flux sheet is unstable to the undular mode of the magnetic buoyancy instability (i.e., the Parker instability), and small vertical velocity perturbations are imposed initially around the midpoint of the magnetic flux sheet to initiate the instability.

As the resistivity model we adopt the anomalous resistivity type model; the resistivity is assumed to be $\eta \propto\left(v_{d}-v_{c}\right)^{2}$, where $v_{d}=j / \rho$ is the drift velocity of the diamagnetic current in the neutral sheet, $j$ is the current density, $\rho$ is the mass density, and $v_{c}$ is the critical velocity above which anomalous resistivity sets in. The effective magnetic Reynolds number $R_{m, e f f}=V_{A} L / \eta \simeq 1000$ when the magnetic reconnection is occurring. Here, $L$ is the length of the current sheet, and $V_{A}$ is the Alfven speed just below the sheet.

\section{RESULTS AND DISCUSSION}

Figure 1 shows a typical example of numerical simulation results. Below we describe the evolutionary features of the results shown in figure 1 in detail.

(1) Emergence of Magnetic Flux: The early stage $(0 \leq t \leq 68.1 \tau=22.7 \mathrm{~min})$ of the emergence of magnetic flux is essentially the same as in previous models (Shibata et al. 1989, Nozawa et al. 1992). The dense loop at the top of the emerging flux may be identified as an arch filament.

(2) Current Sheet Formation: After $t \simeq 68.1 \tau$, the emerging flux enters the coronal level, and a current sheet is created between the emerging flux and the coronal field (see $t=81.7 \tau=27.2 \mathrm{~min}$ ). At this stage, the rise velocity of the magnetic loop is $\simeq 1 C_{s} \simeq 10 \mathrm{~km} \mathrm{~s}^{-1} \simeq 0.1 V_{A}$ and the downflow speed is $\simeq 30-50 \mathrm{~km} \mathrm{~s}^{-1}$, both of which are consistent with observed values for arch filaments (e.g., Chou and Zirin 1988).

(3) Formation of Magnetic Islands: When the loop top reaches a height of $\sim 30 \mathrm{H} \simeq 6000 \mathrm{~km}$ at $t \simeq 88.2 \tau=29.4 \mathrm{~min}$, most of mass in the dense current sheet has fallen, so that the reconnection suddenly starts to create three magnetic islands, which confine cool, dense, chromospheric plasmas. The size of the islands are $\sim 11 H \simeq 11 D$, consistent with the tearing instability, where $D \simeq 1 H$ is the thickness of the current sheet. Since the dense sheet (loop) may correspond to the arch filament, this suggests that the start of a compact flare or an XBP follows the disappearance of an arch filament.

(4) Coalescence of Magnetic Islands: Two of three magnetic islands dynamically coalesce via the coalescence instability (Tajima and Sakai 1989). The cool plasmas in the islands are little heated during the coalescence.

(5) Creation of Hot Plasma in the Current Sheet: During the formation and coalescence of the magnetic islands, hot plasma $\left(T>3 T_{\text {corona }}\right)$ is continuosly created by reconnection in the current sheet. It is remarkable that both cool $\left(T \simeq(1-5) \times 10^{4}\right.$ $\mathrm{K})$ and hot $\left(T>(1-3) \times 10^{6} \mathrm{~K}\right)$ plasmas coexist near the current sheet. Although 
the heat conduction is neglected in this simulation, we expect that the cool plasma in the island would survive even if heat conduction is included, because the magnetic field surrounding the island prevents heat from conducting in to the cool plasma.
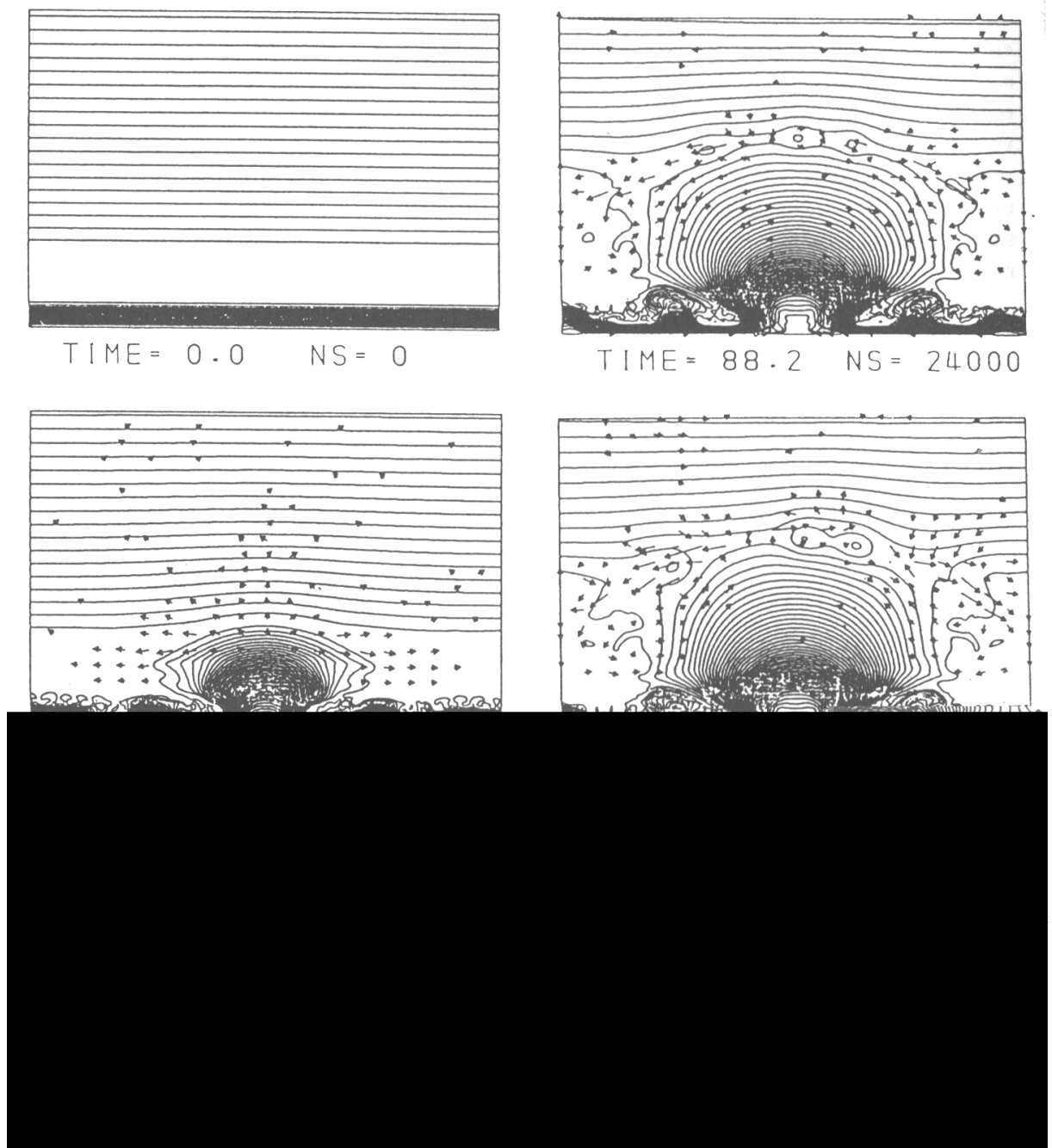

TIME $=81.7 \quad N S=22000$

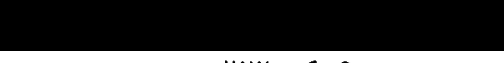

- YNM- 6.0

Fig. 1. Magnetic field lines and velocity vectors in a typical example of magnetic reconnection between emerging flux and the overlying coronal magnetic field. The times are in units of $\tau=H / C_{s} \simeq 20 \mathrm{sec}$, and the horizontal and vertical sizes of the computing box are $x_{\max }=80 H=16000 \mathrm{~km}$ and $z_{\max }+5 H=54 H=10800$ $\mathrm{km}$. The scale of the velocity vector is shown below the frame of time $=96.1$ in units of $C_{s} ; \mathrm{VNM}=6.0$ indicates that the arrow with the length of this line has the velocity of $6.0 C_{s} \simeq 60 \mathrm{~km} \mathrm{~s}^{-1}$. 
(6) Ejection of Cool Magnetic Islands and Ambient Hot Plasma: Both the cool plasma in the islands and the hot plasma in the current sheet are accelerated along the sheet up to $\sim$ Alfven speed $\left(\simeq 50-100 \mathrm{~km} \mathrm{~s}^{-1}\right)$ just below the sheet. The velocity of the cool and hot plasmas is comparable to that of the surges observed in the early stage of the emerging flux region (Kurokawa and Kawai 1993). So we conclude that observed mass ejections (or the jets), as consisting of both hot and cool plasmas, are remarkably well reproduced in our numerical simulations.

(7) Violent Reconnection just after Ejection of a Large Coalesced Island: After a large coalesced island is ejected, the reconnection becomes more violent (at $t / \tau \simeq$ 96.1) than before so that the temperature in the current sheet reaches a maximum value $\left(T_{\max } \sim 10 \times T_{\text {corona }}\right)$. The rate of mass inflow into the current sheet also reaches a maximum. Such violent reconnection seems to be a result of the following process: the rapid ejection of a large island creates an instantaneous "vacuum" in the current sheet, which induces the collapse of the sheet and hence a violent magnetic reconnection. This process may correspond to the impulsive phase of compact flares and/or X-ray bright points.

(8) Fast Shocks: The velocity of the mass ejection along the sheet exceeds the local magnetosonic speed, so that fast shocks are created at both edges of the current sheet. Heating by these fast shocks $\left(T_{s h} \simeq(5-10) \times 10^{5} \mathrm{~K}\right)$ may also account for $\mathrm{UV}$ microflares, compact flares, and/or X-ray bright points.

(9) End of Reconnection: Magnetic reconnection ceases after when $t \simeq 106.9 \tau=$ $35.6 \mathrm{~min}$. Hence the duration of the reconnection is only $\sim 18 \tau \sim 6 \mathrm{~min}$. The total energy released during the reconnection amounts to $10^{28}-10^{29} \mathrm{erg}$, if the size of the current sheet in $y$-direction is $\sim 10^{4} \mathrm{~km}$.

Recent observation by the Yohkoh soft X-ray telescope has revealed many X-ray jets in the solar corona, some of which have been identified with $\mathrm{H} \alpha$ surges (Shibata et al. 1992b, 1993). Such coexistence of $\mathrm{X}$-ray jets and $\mathrm{H} \alpha$ surges is natually explained by our simulation results, i.e., by the simultaneous ejection of cool and hot plasmas discussed in item (6). (See Yokoyama and Shibata 1993 for further study.)

\section{$\underline{\text { REFERENCES }}$}

Chou, D. and Zirin, H.: 1988, Ap. J., 333, 420.

Forbes, T. G. and Priest, E. R.: 1984, Solar Phys., 94, 315.

Kundu, R. and Woodgate, B. (ed.): 1986, Proc. SMM Workshop, Energetic Phenomena on the Sun, NASA Publ. No. 2439, chap. 1

Kurokawa, H. and Kawai, G.: 1993, in these proceedings.

Nozawa, S. et al.: 1992, Ap. J. Suppl., 78, 267.

Shibata, K., Tajima, T., Steinolfson, R. and Matsumoto, R.: 1989, Ap. J., 345, 584.

Shibata, K., Nozawa, S., Matsumoto, R., Sterling, A. C., and Tajima, T.: 1990, Ap. J., $351, \mathrm{~L} 25$.

Shibata, K., Nozawa, S., and Matsumoto, R.: 1992a, Publ. Astron. Soc. Japan, 44, 265.

Shibata, K. et al:: 1992b, Publ. Astron. Soc. Japan, 44, L173.

Shibata, K. et al:: 1993, in these proceedings.

Tajima, T. and Sakai, J-I.: 1989, Sov. J. Plasma Phys., 15, 519.

Yokoyama, T., and Shibata, K.: 1993, Proc. of Fourth Toki conference on Fusion and Astrophysical Plasmas, ESA SP-351, in press. 